\title{
Carrier density modulation and photocarrier transportation of graphene/InSb heterojunction middle-wavelength infrared photodetectors
}

\author{
Shoichiro Fukushima, Masaaki Shimatani, Satoshi Okuda, and \\ Shinpei Ogawa* \\ Mitsubishi Electric Corporation, Advanced Technology R\&D Center, Amagasaki, Hyogo, Japan
}

\begin{abstract}
The photoresponse mechanism of graphene/InSb heterojunction middle-wavelength infrared (MWIR) photodetectors was investigated. The devices comprised a graphene/InSb heterojunction as a carrier-injection region and an insulator region of graphene on tetraethyl orthosilicate (TEOS) for photogating. The MWIR photoresponse was significantly amplified with an increase in the graphene/TEOS cross-sectional area by covering the entire detector with graphene. The graphene-channel dependence of the MWIR photoresponse indicated that the graphene carrier density was modulated by photocarrier accumulation at the TEOS/InSb boundary, resulting in photogating. The dark current of the devices was suppressed by a decrease in the graphene/InSb carrier-injection region and the formation of the heterojunction using an n-type InSb substrate. The results indicate that photocarrier transportation was dominated by the formation of a Schottky barrier at the interface of the graphene/InSb heterojunction and a Fermi-level shift under bias application. The high-responsivity and low-dark-current photoresponse mechanism was attributed to the graphene/InSb heterojunction diode behavior and the photogating effect. The devices combining the aforementioned features had a noise equivalent power of $0.43 \mathrm{pW} / \mathrm{Hz}^{1 / 2}$. The results obtained in our study will contribute to the development of high-performance graphene-based IR image sensors. () The Authors. Published by SPIE under a Creative Commons Attribution 4.0 Unported License. Distribution or reproduction of this work in whole or in part requires full attribution of the original publication, including its DOI. [DOI: 10.1117/1.OE.59.9 .097101]
\end{abstract}

Keywords: graphene; graphene photodetector; Schottky barrier diode; field-effect transistor; infrared sensor; middle-wavelength infrared.

Paper 20200629 received May 27, 2020; accepted for publication Aug. 14, 2020; published online Sep. 7, 2020.

\section{Introduction}

Graphene-based infrared (IR) photodetectors are promising devices that take advantage of the unique optoelectronic properties of graphene such as broadband light absorption,,${ }^{1-3}$ high carrier mobility, ${ }^{4}$ high thermal conductivity, ${ }^{5-7}$ gate-tunable plasmons, ${ }^{8,9}$ and strong nonlinear optical response, ${ }^{10-12}$ as well as its excellent chemical stability. Graphene exhibits broadband light absorption over wavelengths ranging from the ultraviolet to the terahertz region and a fast photoresponse that provides a GHz-order bandwidth. ${ }^{13,14}$ In addition, graphene can be fabricated at a low cost via nontoxic processing, which is more advantageous than quantum-type IR detector materials. However, conventional graphene field-effect transistors (FETs) have drawbacks for IR photodetector applications in which high-photoresponsivity and low-noise characteristics are required to be consistent. The responsivity of graphene devices suffers because a single layer of the graphene absorbs only $2.3 \%$ of light. ${ }^{15}$ In addition, the dark current of conventional graphene FETs is extremely high because of the intrinsic nature of graphene with a zero-bandgap structure. ${ }^{4,16}$ Meanwhile, the dark current should be suppressed to improve signal-to-noise performance. To develop high-performance graphene IR photodetectors, these deficiencies should be improved.

*Address all correspondence to Shinpei Ogawa, E-mail: Ogawa.Shimpei@eb.MitsubishiElectric.co.jp 
We previously investigated responsivity enhancement and dark-current reduction techniques individually using devices equipped with graphene/insulator layers and graphene/semiconductor heterojunction structures. ${ }^{17,18}$ Although various studies have revealed Schottky-barrier formation and carrier transport phenomenon in graphene and semiconductors including $\mathrm{Si},{ }^{19-27} \mathrm{Ge},{ }^{28,29}$ $\mathrm{GaAs},{ }^{30-32} \mathrm{CdSe},{ }^{33,34} \mathrm{SiC},{ }^{35,36}$ and $\mathrm{GaN},{ }^{37,38}$ it is not clear how the extraordinary high responsivity is obtained in the heterojunction structure. We have proved the graphene/insulator layer region underwent photogating, ${ }^{3,17,39-44}$ which is one of the most effective responsivity enhancement candidates among possible techniques, such as pn junctions; ${ }^{45}$ turbostacking of graphene ${ }^{40}$ plasmonic metamaterial absorbers; ${ }^{46-48}$ the addition of photosensitizers including $\mathrm{MoS}_{2},{ }^{49,50} \mathrm{ZnO},{ }^{51,52}$ organic semiconductor, ${ }^{53}$ and quantum dots $;{ }^{54-57}$ and optical waveguides. ${ }^{58}$ Photogating modulates the surface carrier density of graphene by locating a photosensitizer in the vicinity of the graphene. This multiplies the photocarrier transport from the graphene/semiconductor heterojunction region. Moreover, the dark current can be suppressed by adjusting the applied voltage. However, the detailed mechanism of this structure has not yet been investigated.

Here, we report on a detailed mechanism of the devices and design principle of structures to improve the responsivity and dark-current characteristics in parallel. InSb, which is well known photomaterial for middle-wavelength IR (MWIR) detection, was applied. The responsivity enhancement was assessed by comparing devices entirely and partially covered with graphene. The graphene-channel dependence of the MWIR photoresponse enhancement was investigated to assess the photogating. A low-dark-current bias region was compared between devices with distinct graphene/InSb heterojunction areas and dopants of InSb substrate. The MWIR photoresponse performance of the devices that combined the features above was evaluated.

\section{Device Fabrication and Assessment}

Figure 1 shows a schematic of the graphene/InSb heterojunction photodetector. The devices consist of a graphene and p/n-doped InSb heterojunction and a graphene/tetraethyl orthosilicate (TEOS) region. A $600-\mu$ m-thick InSb substrate with a 100-nm-thick TEOS insulator layer was prepared. The carrier concentrations of the $\mathrm{p} / \mathrm{n}$-doped substrate are $2 \times 10^{14-15}$ and 1 to $3 \times 10^{15} \mathrm{~cm}^{3}$, respectively. The drain electrode consisted of 10 -nm-thick $\mathrm{Cr}$ and 50 -nm-thick $\mathrm{Au}$ layers and was sputtered on the TEOS layer. The TEOS layer on the center region of the devices was etched using buffered hydrogen fluoride to form a graphene/InSb region. Graphene was fabricated by chemical vapor deposition and was transferred onto the surface of the devices using a conventional graphene-transfer method. ${ }^{59,60}$ The graphene channel was formed through a conventional photolithography process and oxygen plasma etching. To assess the graphene cross-sectional area dependence of the MWIR photoresponse enhancement, the devices were entirely or partially covered with graphene, as shown in Figs. 1(b) and 1(c).

Figure 2 shows the Raman spectrum obtained for the graphene channel using a $512 \mathrm{~nm}$ excitation laser. The spectrum has the typical characteristics of graphene, including a $\mathrm{G}$ peak at $1580 \mathrm{~cm}^{-1}$ and a D peak at $2700 \mathrm{~cm}^{-1}$, which corresponds to the bond stretching and secondorder breathing modes of $\mathrm{sp}^{2}$ carbon atoms, respectively. The spectrum indicates that a monolayer graphene channel was successfully formed. ${ }^{61,62}$

The photoresponse characteristics were investigated by current measurement. The devices were set in a vacuum probe chamber with a cooler at $10^{-3} \mathrm{~Pa}$ and $77 \mathrm{~K}$. The backside of the
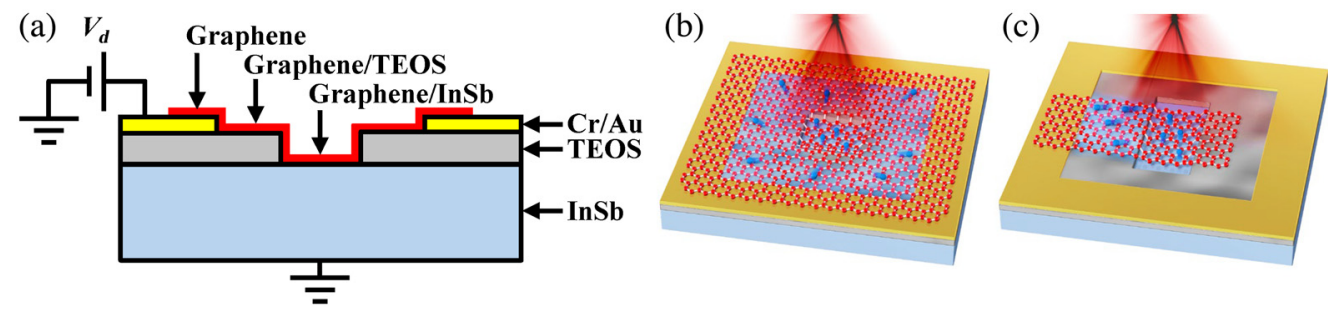

Fig. 1 Schematic illustration of (a) graphene-based photodetector design and MWIR photoresponse in the devices (b) entirely or (c) partially covered with graphene. 


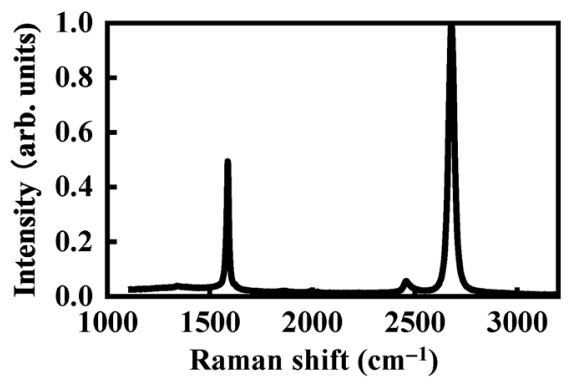

Fig. 2 Raman spectrum of graphene channel in the TEOS insulator layer of the devices in Fig. 1(b).

substrate was electrically grounded. Current measurements and voltage application were conducted using a device analyzer (B1500A, Keysight). A quantum cascade laser (QD4550CM1, Thorlabs) with a wavelength of $4.6 \mu \mathrm{m}$ was used as the light source.

\section{Results and Discussion}

\subsection{Basic Device Characteristics}

Figure 3(a) shows the drain current $\left(I_{d}\right)$ response of the devices using a drain voltage $\left(V_{d}\right)$ of $0 \mathrm{~V}$. The devices were exposed to the $4.6 \mu \mathrm{m}$ laser with a $2.0 \mathrm{~s}$ irradiation cycle $(0.8 \mathrm{~s}$ on and $1.2 \mathrm{~s}$ off). The devices exhibited a definite photoresponse by modulation with an $I_{d}$ of $10.37 \pm$ $0.15 \mu \mathrm{A}$ as the photocurrent with a base $I_{d}$ of $0.86 \mu \mathrm{A}$. Figure 3(b) shows the current-bias characteristics of the devices under various laser powers. The photocurrent showed a linear increase with variation of the laser light power and $V_{d}$ application. The maximum MWIR light responsivity of the devices was calculated to be $4.68 \mathrm{~A} / \mathrm{W}$ at a $V_{d}$ of $0.5 \mathrm{~V}$. We also confirmed that the devices exhibited photoresponses under visible, near-IR, and MWIR around 3 to $5 \mu \mathrm{m}$.

\subsection{Graphene Channel Dependence of Photogating}

The MWIR photoresponse of the devices was investigated, and Fig. 4 shows a comparison of the photocurrent characteristics of entirely and partially graphene-covered structures. The graphene/ InSb contact region was $50 \times 50 \mu \mathrm{m}^{2}$ in both devices. The entirely graphene-covered device indicated a significant increase in the photocurrent, where the maximum photocurrent at a $V_{d}$ of $0.5 \mathrm{~V}$ reached $67.63 \pm 4.03 \mu \mathrm{A}$, whereas that of the partially graphene-covered device exhibited a maximum photocurrent of $9.44 \pm 0.23 \mu \mathrm{A}$.

The photoresponse of the devices is mainly affected by the graphene/TEOS region because the photogating occurs in this region. Under positive $V_{d}$ application, photogenerated electrons and holes are separated in the p-InSb photosensitizer, and electrons in the vicinity of the graphene/InSb interface are injected into the graphene. On the other hand, photoelectrons excited in the InSb under the graphene/TEOS contact region accumulate at the TEOS/InSb interface owing
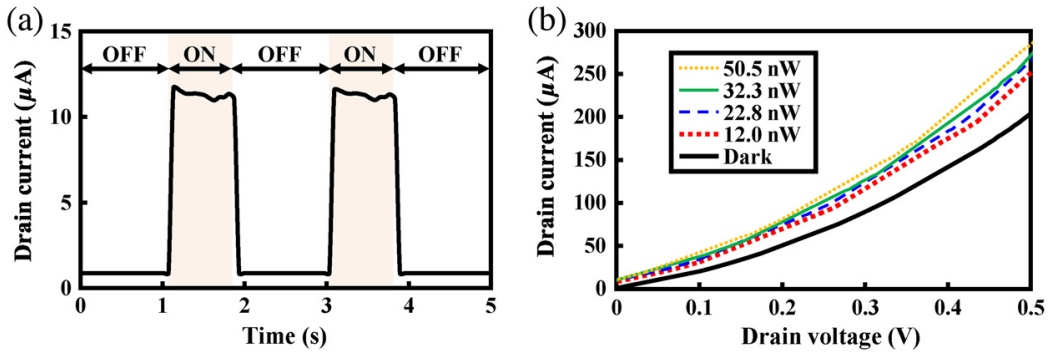

Fig. 3 Photoresponse characteristics of the devices under $4.6 \mu \mathrm{m}$ light irradiation. (a) Drain current response of the devices under pulsed laser irradiation at $V_{d}$ of $0 \mathrm{~V}$. (b) Drain current-voltage characteristics of the devices under various irradiation light powers. 

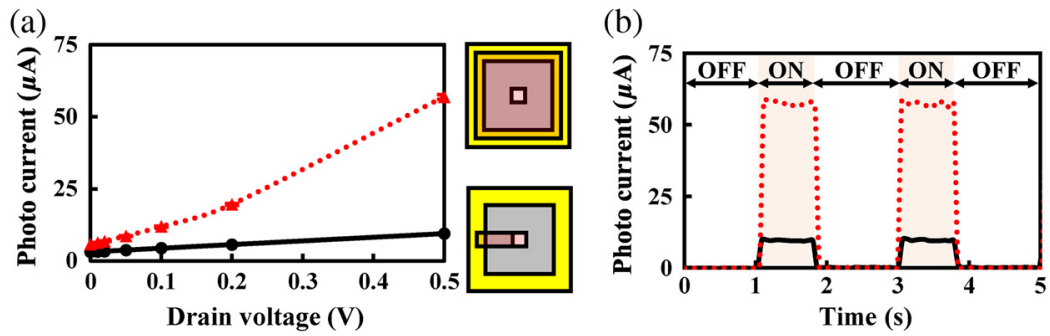

Fig. 4 Comparison of photoresponse in devices with different graphene channel shapes. (a) Photocurrent as a function of $V_{d}$, with graphene channel connecting graphene/InSb contact entirely (red dotted) and partially (black solid) graphene coverage. Inset: schematic of respective devices. (b) Photocurrent-time characteristics of devices at $V_{d}$ of $0.5 \mathrm{~V}$.

to the depletion layer formed by $V_{d}$ application, which changes the graphene's surface carrier density. As a result, the photocurrent of the devices can be amplified. This phenomenon is referred to as photogating. The remaining TEOS region can provide additional photogating by the photocarriers generated in the TEOS/InSb depletion layer, although it may be less effective for photoresponse enhancement because the carrier diffusion distance of the InSb is short, at around a few micrometers, ${ }^{41}$ and the effective photocarrier region is situated only in the vicinity of the graphene/TEOS region. The TEOS layer may also decrease the MWIR incident light power that penetrates to the $\mathrm{InSb}$ substrate. TEOS- $\mathrm{SiO}_{2}$ has an absorption peak around 9 to $10 \mu \mathrm{m}$; therefore, the effect of this decrease is negligible. The results show that photogating in the graphene/TEOS region plays a dominant role in the MWIR photoresponse.

To clarify the influence of the graphene/TEOS region on the responsivity enhancement, the MWIR photoresponse characteristics obtained with different graphene/TEOS cross-sectional areas were compared. Figure 5(a) shows a schematic diagram of a device structure. In the region with graphene, $S_{\mathrm{CH}}, L$, and $W$ are defined as the graphene/TEOS region, length, and width, respectively. As shown in Fig. 5(b), the photoresponse increased with an increase in $S_{\mathrm{CH}}$. Next, the effects of the graphene channel length $L$ with a fixed $W$ of $100 \mu \mathrm{m}$ and $W$ with a fixed $L$ of $200 \mu \mathrm{m}$ were investigated. Figures 5(c) and 5(d) show that the photocurrent increased as $L$ and $W$ increased.

The photoresponse in graphene FETs with photogating is dependent on the graphene-channel aspect ratio because the devices operate under the same principles as metal-oxide-semiconductor

(a)

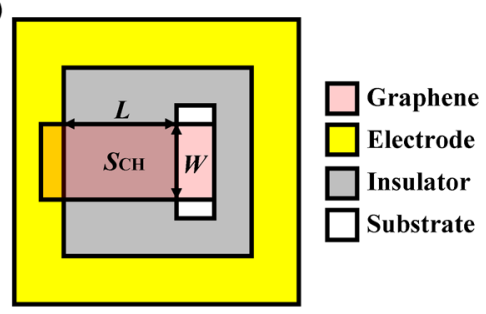

(c)

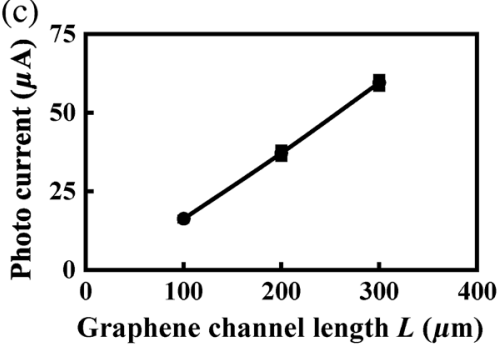

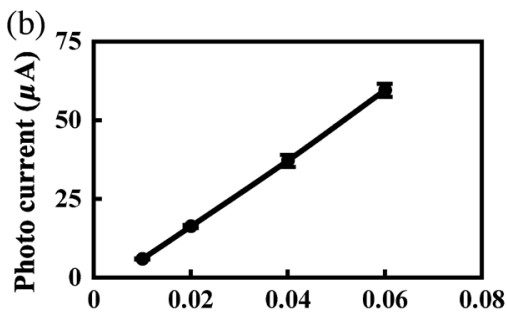

Graphene channel area $S_{\mathrm{CH}}\left(\mathrm{mm}^{2}\right)$

(d)

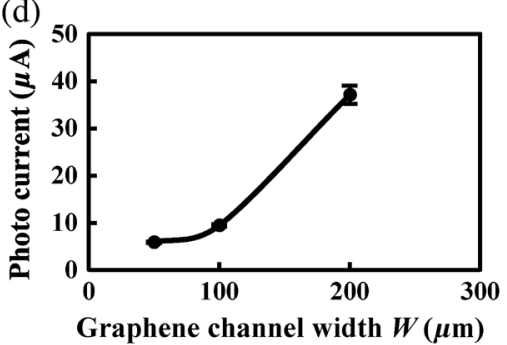

Fig. 5 (a) Schematic of graphene channel in the devices, indicating each symbolic character $S_{C H}$, $W$, and $L$. (b)-(d) Photocurrent characteristics in graphene photodetectors for various graphenechannel cross-sectional areas (b) $S_{\mathrm{CH}}$, (c) lengths $L$, and (d) widths $W$. 
FETs. ${ }^{42,44}$ By contrast, the devices with graphene/InSb heterojunction structures exhibit greater photocurrent when the graphene-channel $W, L$, and $S_{\mathrm{CH}}$ increase. An entirely covered graphene channel enables the injected photocarriers in the graphene/InSb heterojunction region to spread to the drain electrode in all directions. This increases the effective region of the photogating, which multiplies the photocurrent. In addition, the photocurrent is nonlinearly increased with $W$. Although an increase in $L$ increases only the graphene/TEOS region for the photogating effect, an increase in $W$ increases both the graphene/TEOS region and graphene/InSb heterojunction region. The heterojunction region size does not affect the graphene/InSb Schottky barrier height but changes the flow rate of the photocarriers between graphene and InSb. Both the increase in photocarriers injected into graphene and the improvement of the responsivity due to photogating cause a nonlinear increase.

\subsection{Dark-Current Reduction}

Next, the dark-current dependence on the device structures was investigated. Figures 6(a) and 6(b) show the $V_{d}$-dependent dark-current characteristics with different graphene/InSb heterojunction cross-sectional areas and dopant types of the InSb substrate. The devices were fabricated to be entirely covered with a graphene channel, with $S_{\mathrm{CH}}$ and the graphene/n-InSb contact region $\left(S_{\mathrm{CI}}\right)$ set as $50 \times 50 \mu \mathrm{m}^{2}, 100 \times 100 \mu \mathrm{m}^{2}$, and $200 \times 200 \mu \mathrm{m}^{2}$, respectively, as shown in Fig. 6(c). The maximum dark current decreased with decreasing $S_{\mathrm{CI}}$, and the bias region of low dark current within $5 \mu \mathrm{A}$ expanded from 62 to 73 and $85 \mathrm{mV}$ in graphene/p-InSb devices and from 54 to 708 and $711 \mathrm{mV}$ in graphene/n-InSb devices according to decreasing $S_{\mathrm{CI}}$. These results indicate that $S_{\mathrm{CI}}$ significantly affects the photoresponse and operating characteristics of both dopant types.

The dark-current behavior was investigated using a band model of a graphene/InSb heterojunction, as shown in Figs. 6(d) and 6(e). A reverse bias decreases the Schottky barrier height $\left(\Phi_{B}\right)$, which corresponds to the work function difference between the Fermi level of graphene and the valence band of $\mathrm{n}-\mathrm{InSb}$, and causes a leak current for both graphene/p-InSb and graphene/n-InSb structures. The work function of InSb is around $4.77 \mathrm{eV}^{63}$ and is very close to that of the graphene at around 4.5 to $5.0 \mathrm{eV}^{64-66}$ Since the work function of graphene is strongly affected by the surface condition ${ }^{45,67}$ in which the adhesion of moisture in the atmosphere or residue causes hole doping ${ }^{68}$ the Fermi level $E_{F}$ of graphene is lowered from the neutral point. This lower shift of the Fermi level in graphene increases $\Phi_{B}$ and suppresses the dark-current leak at the graphene/n-InSb heterojunction.
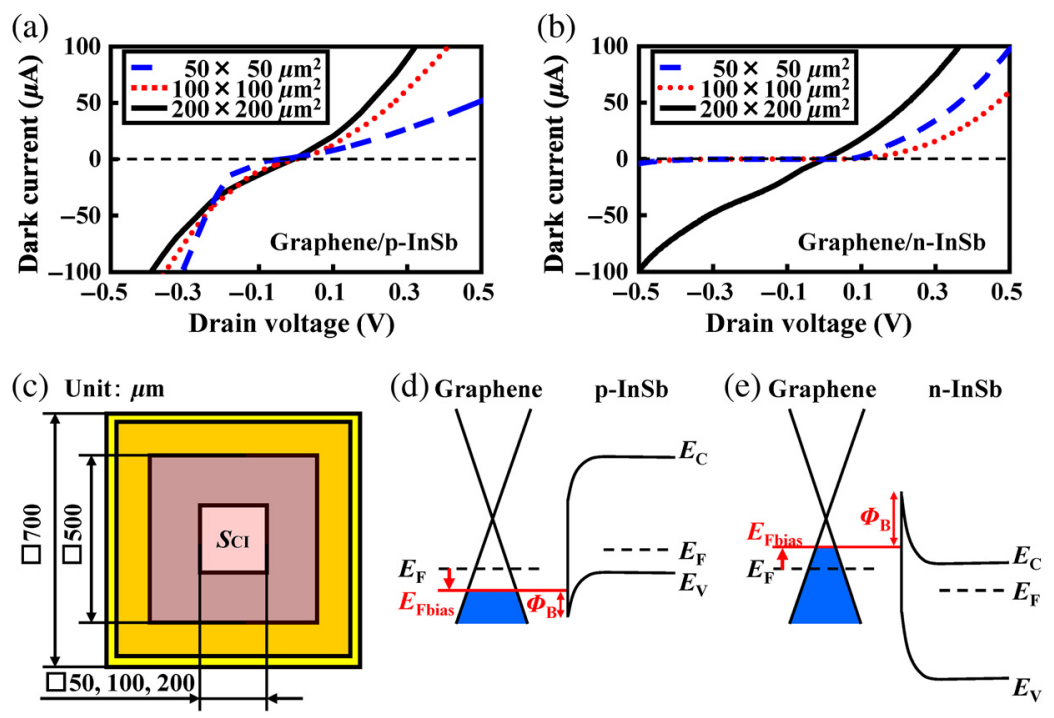

Fig. 6 (a), (b) Dark-current characteristics in (a) graphene/p-InSb and (b) graphene/n-InSb devices with various carrier-injection areas $S_{\mathrm{Cl}}$. (c) Schematic illustration of the devices. Energy band diagram of $(d)$ graphene/p-InSb and (e) n-InSb heterojunction under application of reverse bias. 
(a)

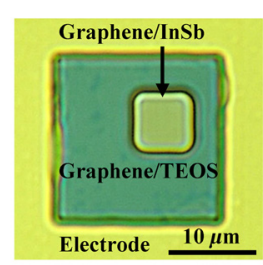

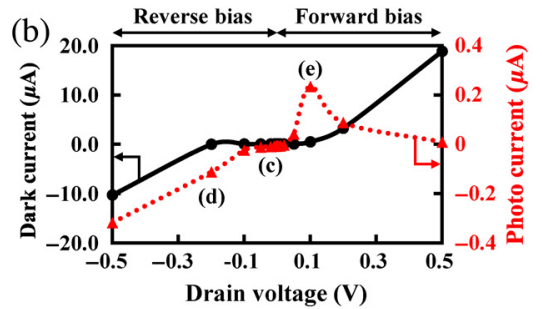
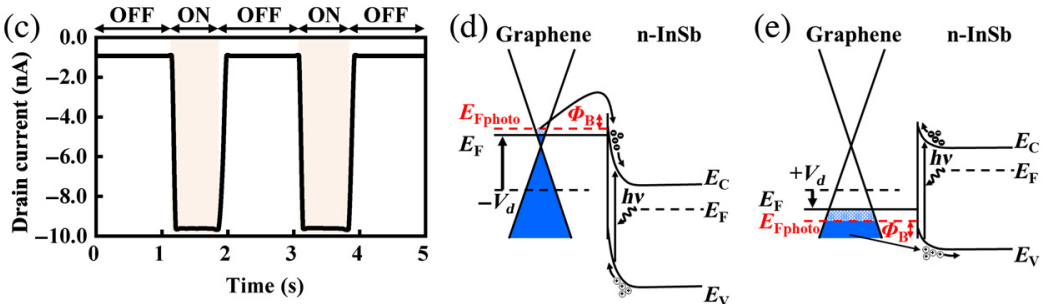

Fig. 7 Photoresponse characteristics in graphene/n-InSb photodetectors. (a) Optical microscopy image of the device. (b) Dark current (black, solid) and photocurrent (red, dotted) voltage characteristics of the devices. (c) Photocurrent-time characteristics of the devices under reverse bias application of $-1 \mathrm{mV}$. (d), (e) Schematic of energy band diagram of the devices under (d) reverse bias of $-0.2 \mathrm{~V}$ and $(\mathrm{e})$ forward bias of $0.1 \mathrm{~V}$ as indicated in (b).

\subsection{Performance Evaluation of Improved Structured Device}

The MWIR photoresponse performance of the devices that combines the aforementioned features was evaluated. The devices were fabricated as fully covered with a graphene channel of $20 \times 20 \mu \mathrm{m}^{2}$ as the MWIR light irradiation area, and the graphene/n-InSb contact region $S_{\mathrm{CI}}$ was decreased to $7 \times 7 \mu \mathrm{m}^{2}$, as shown in Fig. $7\left(\right.$ a). Figure 7 (b) shows the $V_{d}$-dependent dark/ photocurrent characteristics of the devices. The devices exhibited diode characteristics, and the dark current was suppressed within $5 \mu \mathrm{A}$ in a bias region of -200 to $200 \mathrm{mV}$ and within $1 \mathrm{nA}$ in a bias region of -2.5 to $27.7 \mathrm{mV}$. The devices exhibited negative photocurrent in a negative $V_{d}$ region and positive photocurrent in a positive $V_{d}$ region under $6.4 \mathrm{~mW} / \mathrm{cm}^{2}$ MWIR light irradiation. Figure 7(c) shows the MWIR performance of the devices with the dark current of $-0.96 \mathrm{nA} \pm 0.58 \mathrm{pA}$ and photocurrent of $-8.67 \pm 0.08 \mathrm{nA}$ at a $V_{d}$ of $-1 \mathrm{mV}$, which corresponds to a noise equivalent power (NEP) of $0.43 \mathrm{pW} / \mathrm{Hz}^{1 / 2}$. The performance of the graphene photodetectors is more dependent on the device fabrication process and the graphene synthesis method than on the device structure. The NEP value was significantly improved from $94.5 \mathrm{nW} / \mathrm{Hz}^{1 / 2}$ in our previous work, ${ }^{17}$ where the graphene and other device elements were prepared in the same manner.

The devices exhibited a high responsivity that exceeded $100 \%$ of the external quantum efficiency under a large bias application. The responsivity reached $6.14 \mathrm{~A} / \mathrm{W}$ at $V_{d}$ at $-0.2 \mathrm{~V}$ and $12.6 \mathrm{~A} / \mathrm{W}$ at $V_{d}$ at $0.1 \mathrm{~V}$. Figures 7(d) and 7(e) show band models of the graphene/n-InSb heterojunction under reverse and forward bias. Under reverse-bias negative $V_{d}$ application, the Fermi level is upshifted in the graphene. $\Phi_{B}$ is further decreased with the upshift of the Fermi level by photogenerated electrons in the graphene from $E_{F}$ to $E_{F \text { photo }}$. The photogenerated electrons can obtain enough energy to generate an avalanche multiplication process at the depletion layer formed in the graphene/n-InSb heterojunction. Since the carrier transportation has not alienated the negative $V_{d}$ region, the dark current is also increased with the photocurrent. By contrast, a positive $V_{d}$ application and photogenerated holes decrease $\Phi_{B}$, and the photogenerated electrons in the InSb and the holes in graphene are transported between each other. A further increase of positive $V_{d}$ promotes the recombination of the photogenerated electrons and injected holes in $\mathrm{n}-\mathrm{InSb}$, and the photocurrent decreases. The results obtained here indicate that the high performance of the devices stands on the formation of Schottky barriers at the interface of the graphene/n-InSb heterojunction and photocarrier transportation. 


\section{Conclusion}

We investigated graphene/InSb heterojunction MWIR photodetectors that can achieve high responsivity and low-dark-current characteristics. The MWIR photoresponse of the devices indicated that the injected photocarriers from the substrate to graphene were amplified by the photogating induced in the graphene/TEOS region. The photocurrent characteristics for various graphene channel sizes indicated that the photocurrent changes linearly with the cross-sectional area of the graphene/TEOS region, in which the photogating contributes large photocurrent modulation. In addition, the graphene shape entirely covers the device area, thus enhancing the photogating. It was also demonstrated that the graphene-InSb substrate heterojunction region has a significant influence on the dark-current performance, and the dark current was suppressed by a decrease in the graphene/InSb carrier-injection region and formation of the heterojunction using an n-type InSb substrate. The voltage-dependent current characteristics indicated that photocarrier transportation was dominated by the formation of a Schottky barrier at the graphene/ InSb heterojunction and a Fermi-level shift of the graphene under bias application. The devices that combined these features exhibited NEP of $0.43 \mathrm{pW} / \mathrm{Hz}^{1 / 2}$. The results obtained in this study will contribute to the development of high-performance graphene-based IR image sensors.

\section{Acknowledgments}

This work was supported by the Innovative Science and Technology Initiative for Security Grant No. JPJ004596, ATLA, Japan. There are no conflicts of interest to declare.

\section{References}

1. G. Pirruccio et al., "Coherent and broadband enhanced optical absorption in graphene," $A C S$ Nano 7(6), 4810-4817 (2013).

2. C.-H. Liu et al., "Graphene photodetectors with ultra-broadband and high responsivity at room temperature," Nat. Nanotechnol. 9(4), 273-278 (2014).

3. S. Ogawa et al., "Broadband photoresponse of graphene photodetector from visible to longwavelength infrared wavelengths," Opt. Eng. 58(5), 057106 (2019).

4. K. S. Novoselov et al., "Electric field effect in atomically thin carbon films," Science 306(5696), 666-669 (2004).

5. J. C. Song et al., "Hot carrier transport and photocurrent response in graphene," Nano Lett. 11(11), 4688-4692 (2011).

6. D. Sun et al., "Ultrafast hot-carrier-dominated photocurrent in graphene," Nat. Nanotechnol. 7(2), 114-118 (2012).

7. X. Cai et al., "Sensitive room-temperature terahertz detection via the photothermoelectric effect in graphene," Nat. Nanotechnol. 9(10), 814-819 (2014).

8. J. Chen et al., "Optical nano-imaging of gate-tunable graphene plasmons," Nature 487(7405), 77-81 (2012).

9. B. Yao et al., "Broadband gate-tunable terahertz plasmons in graphene heterostructures," Nat. Photonics 12(1), 22-28 (2018).

10. E. Hendry et al., "Coherent nonlinear optical response of graphene," Phys. Rev. Lett. 105(9), 097401 (2010).

11. S. Shareef, Y. S. Ang, and C. Zhang, "Room-temperature strong terahertz photon mixing in graphene," J. Opt. Soc. Am. B 29(3), 274-279 (2012).

12. K. J. A. Ooi et al., "Electronic scattering of graphene plasmons in the terahertz nonlinear regime," IEEE J. Sel. Top. Quantum Electron. 23(4), 1-6 (2017).

13. F. Xia et al., "Ultrafast graphene photodetector," Nat. Nanotechnol. 4(12), 839-843 (2009).

14. A. Urich, K. Unterrainer, and T. Mueller, "Intrinsic response time of graphene photodetectors," Nano Lett. 11(7), 2804-2808 (2011).

15. K. F. Mak et al., "Measurement of the optical conductivity of graphene," Phys. Rev. Lett. 101(19), 196405 (2008).

16. K. S. Novoselov et al., "Two-dimensional gas of massless Dirac fermions in graphene," Nature 438(7065), 197-200 (2005). 
17. S. Fukushima et al., "Low dark current and high-responsivity graphene mid-infrared photodetectors using amplification of injected photo-carriers by photo-gating," Opt. Lett. 44(10), 2598-2601 (2019).

18. S. Fukushima et al., "Middle wavelength infrared graphene photodetectors with low darkcurrent and high responsivity," Proc. SPIE 11407, 1140718 (2020).

19. T. Feng et al., "Graphene based Schottky junction solar cells on patterned silicon-pillar-array substrate," Appl. Phys. Lett. 99(23), 233505 (2011).

20. C.-C. Chen et al., "Graphene-silicon Schottky diodes," Nano Lett. 11(5), 1863-1867 (2011).

21. Y. An et al., "Metal-semiconductor-metal photodetectors based on graphene/p-type silicon Schottky junctions," Appl. Phys. Lett. 102(1), 013110 (2013).

22. P. Lv et al., "High-sensitivity and fast-response graphene/crystalline silicon Schottky junction-based near-IR photodetectors," IEEE Electron Device Lett. 34(10), 1337-1339 (2013).

23. M. Amirmazlaghani et al., "Graphene-Si Schottky IR detector," IEEE J. Quantum Electron. 49(7), 589-594 (2013).

24. C. Xie et al., "High-efficiency, air stable graphene/Si micro-hole array Schottky junction solar cells," J. Mater. Chem. A 1(48), 15348 (2013).

25. Y. Song et al., "Role of interfacial oxide in high-efficiency graphene-silicon Schottky barrier solar cells," Nano Lett. 15(3), 2104-2110 (2015).

26. M. Serry et al., "Nanostructured graphene-Schottky junction low-bias radiation sensors," Sens. Actuators A 232, 329-340 (2015).

27. M. Casalino et al., "Vertically illuminated, resonant cavity enhanced, graphene-silicon Schottky photodetectors," ACS Nano 11(11), 10955-10963 (2017).

28. L.-H. Zeng et al., "Monolayer graphene/germanium schottky junction as high-performance self-driven infrared light photodetector," ACS Appl. Mater. Interfaces 5(19), 9362-9366 (2013).

29. K. E. Chang et al., "High-responsivity near-infrared photodetector using gate-modulated graphene/germanium Schottky junction," Adv. Electron. Mater. 5(6), 1800957 (2019).

30. W. Jie, F. Zheng, and J. Hao, "Graphene/gallium arsenide-based Schottky junction solar cells," Appl. Phys. Lett. 103(23), 233111 (2013).

31. L.-B. Luo et al., "Near-infrared light photovoltaic detector based on GaAs nanocone array/ monolayer graphene Schottky junction," Adv. Funct. Mater. 24(19), 2794-2800 (2014).

32. X. Li et al., "Graphene/h-BN/GaAs sandwich diode as solar cell and photodetector," Opt. Express 24(1), 134-145 (2016).

33. Y. Ye et al., "A simple and scalable graphene patterning method and its application in CdSe nanobelt/graphene Schottky junction solar cells," Nanoscale 3(4), 1477-1481 (2011).

34. Z. Gao et al., "Flexible solar cells based on CdSe nanobelt/graphene Schottky junctions," J. Mater. Chem. C 3(17), 4511-4514 (2015).

35. S. Shivaraman et al., "Schottky barrier inhomogeneities at the interface of few layer epitaxial graphene and silicon carbide," Appl. Phys. Lett. 100(18), 183112 (2012).

36. H. Li et al., "Atomic-scale tuning of graphene/cubic SiC Schottky junction for stable lowbias photoelectrochemical solar-to-fuel conversion," ACS Nano 14(4), 4905-4915 (2020).

37. S. Tongay et al., "Graphene/GaN Schottky diodes: stability at elevated temperatures," Appl. Phys. Lett. 99(10), 102102 (2011).

38. S. Kim et al., "Graphene-GaN Schottky diodes," Nano Res. 8(4), 1327-1338 (2015).

39. M. Shimatani et al., "Giant Dirac point shift of graphene phototransistors by doped silicon substrate current," AIP Adv. 6(3), 035113 (2016).

40. M. Shimatani et al., "High-responsivity turbostratic stacked graphene photodetectors using enhanced photogating," Appl. Phys. Express 12(12), 122010 (2019).

41. S. Fukushima et al., "High responsivity middle-wavelength infrared graphene photodetectors using photo-gating," Appl. Phys. Lett. 113(6), 061102 (2018).

42. S. Fukushima et al., "High-responsivity graphene infrared photodetectors using photogating effect," Proc. SPIE 11002, 1100223 (2019).

43. M. Shimatani et al., "Enhanced photogating via pyroelectric effect induced by insulator layer for high-responsivity long-wavelength infrared graphene-based photodetectors operating at room temperature," Appl. Phys. Express 12(2), 025001 (2019). 
44. S. Fukushima et al., "Photogating for small high-responsivity graphene middle-wavelength infrared photodetectors," Opt. Eng. 59(3), 037101 (2020).

45. M. Shimatani et al., "Photocurrent enhancement of graphene phototransistors using $\mathrm{p}-\mathrm{n}$ junction formed by conventional photolithography process," Jpn. J. Appl. Phys. 55(11), 110307 (2016).

46. S. Ogawa, D. Fujisawa, and M. Ueno, "Effect of graphene on plasmonic metasurfaces at infrared wavelengths," AIP Adv. 3(11), 112127 (2013).

47. S. Ogawa et al., "Graphene on metal-insulator-metal-based plasmonic metamaterials at infrared wavelengths," Opt. Express 26(5), 5665-5674 (2018).

48. M. Shimatani et al., "Multispectral graphene infrared photodetectors using plasmonic metasurfaces," Proc. SPIE 11002, 1100224 (2019).

49. P. Vabbina et al., "Highly sensitive wide bandwidth photodetector based on internal photoemission in CVD grown p-Type MoS2/graphene Schottky junction," ACS Appl. Mater. Interfaces 7(28), 15206-15213 (2015).

50. H. Xu et al., "High detectivity and transparent few-layer MoS2/glassy-graphene heterostructure photodetectors," Adv. Mater. 30(13), 1706561 (2018).

51. Q. Liu et al., "Transfer-free and printable graphene/ZnO-nanoparticle nanohybrid photodetectors with high performance," J. Mater. Chem. C 5(26), 6427-6432 (2017).

52. B. Nie et al., "Monolayer graphene film on $\mathrm{ZnO}$ nanorod array for high-performance Schottky junction ultraviolet photodetectors," Small 9(17), 2872-2879 (2013).

53. E. H. Huisman et al., "High gain hybrid graphene-organic semiconductor phototransistors," ACS Appl. Mater. Interfaces 7(21), 11083-11088 (2015).

54. A. V. Klekachev et al., "Electron accumulation in graphene by interaction with optically excited quantum dots," Phys. E 43(5), 1046-1049 (2011).

55. C. O. Kim et al., "High-performance graphene-quantum-dot photodetectors," Sci. Rep. 4, 5603 (2015).

56. G. Konstantatos et al., "Hybrid graphene-quantum dot phototransistors with ultrahigh gain," Nat. Nanotechnol. 7, 363 (2012).

57. A. A. Bessonov et al., "Compound quantum dot-perovskite optical absorbers on graphene enhancing short-wave infrared photodetection," ACS Nano 11(6), 5547-5557 (2017).

58. J. Wang et al., "High-responsivity graphene-on-silicon slot waveguide photodetectors," Nanoscale 8(27), 13206-13211 (2016).

59. J. W. Suk et al., "Transfer of CVD-grown monolayer graphene onto arbitrary substrates," ACS Nano 5(9), 6916 (2011).

60. K. Uemura, T. Ikuta, and K. Maehashi, "Turbostratic stacked CVD graphene for highperformance devices," Jpn. J. Appl. Phys. 57(3), 030311 (2018).

61. A. C. Ferrari et al., "Raman spectrum of graphene and graphene layers," Phys. Rev. Lett. 97(18), 187401 (2006).

62. S. Heeg et al., "Polarized plasmonic enhancement by $\mathrm{Au}$ nanostructures probed through Raman scattering of suspended graphene," Nano Lett. 13(1), 301-308 (2013).

63. G. W. Gobeli and F. G. Allen, "Photoelectric properties of cleaved GaAs, GaSb, InAs, and InSb surfaces; comparison with Si and Ge," Phys. Rev. 137(1A), A245-A254 (1965).

64. S. M. Song, J. H. Bong, and B. J. Cho, "Work function tuning of metal/graphene stack electrode," Appl. Phys. Lett. 104(8), 083512 (2014).

65. Y. Shi et al., "Work function engineering of graphene electrode via chemical doping," ACS Nano 4(5), 2689-2694 (2010).

66. T. Filleter et al., "Local work function measurements of epitaxial graphene," Appl. Phys. Lett. 93(13), 133117 (2008).

67. X. Zhang et al., "High-efficiency graphene/Si nanoarray Schottky junction solar cells via surface modification and graphene doping," J. Mater. Chem. A 1(22), 6593 (2013).

68. N. Liu et al., "Large-area, transparent, and flexible infrared photodetector fabricated using $\mathrm{P}-\mathrm{N}$ junctions formed by N-doping chemical vapor deposition grown graphene," Nano Lett. 14(7), 3702-3708 (2014).

Biographies of the authors are not available. 